\title{
ON THE SIGNIFICANCE OF THE EXCESS NUMBER OF STRONG Mg II ABSORBERS OBSERVED TOWARD GAMMA-RAY BURSTS
}

\author{
Sharon Rapoport ${ }^{1}$, Christopher A. Onken $^{1}$, J. Stuart B. Wyithe ${ }^{2}$, Brian P. Schmidt ${ }^{1}$, and Anders O. Thygesen ${ }^{3}$ \\ ${ }^{1}$ Research School of Astronomy and Astrophysics, The Australian National University, Canberra 2611, Australia \\ ${ }^{2}$ School of Physics, University of Melbourne, Parkville, Victoria 3010, Australia \\ ${ }^{3}$ Zentrum für Astronomie der Universität Heidelberg, Landessternwarte, Königstuhl 12, D-69117 Heidelberg, Germany \\ Received 2012 October 22; accepted 2013 February 1; published 2013 March 4
}

\begin{abstract}
The number of strong (equivalent width $>1 \AA$ ) Mg II absorbers observed toward gamma-ray bursts (GRBs) has been found to be statistically larger than the number of strong absorbers toward quasi-stellar objects (QSOs). We formalize this "Mg II problem" and present a detailed explanation of the statistical tools required to assess the significance of the discrepancy. We find that the problem exists at the $4 \sigma$ level for GRBs with high-resolution spectra. It has been suggested that the discrepancy can be resolved by the combination of a dust obscuration bias toward QSOs, and a strong gravitational lensing bias toward GRBs. We investigate one of the two most probable lensed GRBs that we presented in our previous work (GRB020405) and find that it is not strongly gravitationally lensed, constraining the percentage of lensed GRBs to be $<35 \%(2 \sigma)$. Dust obscuration of QSOs has been estimated to be a significant effect with dusty $\mathrm{Mg}$ II systems removing $\sim 20 \%$ of absorbed objects from flux-limited QSO samples. We find that if $\sim 30 \%$ of the strong Mg II systems toward QSOs are missing from the observed samples, then GRBs and QSOs would have comparable numbers of absorbers per unit redshift. Thus, strong gravitational lensing bias is likely to make only a modest contribution to solving the Mg II problem. However, if the dust obscuration bias has been slightly underestimated, the $\mathrm{Mg}$ II problem would no longer persist.
\end{abstract}

Key words: gamma-ray burst: general - gamma-ray burst: individual (GRB020405) - gravitational lensing: strong

Online-only material: color figures

\section{INTRODUCTION}

The number of strong $\mathrm{Mg}$ II absorbers per unit redshift along gamma-ray burst (GRB) lines of sight (LOS) has been found to be statistically different than the rate toward quasi-stellar objects (QSOs). Prochter et al. (2006b, hereafter P06) found 14 strong absorbers along the LOS to 14 GRBs, and Vergani et al. (2009, hereafter V09) increased the sample and found 22 strong absorbers along the LOS to 26 GRBs. In contrast, studies of QSOs have found $\mathrm{Mg}$ II absorbers in only $\sim 25 \%$ of sight lines (Prochter et al. 2006a). Both being high-redshift beacons, GRBs and QSOs are expected to have similar LOS through the cosmos, and explaining the preponderance of $\mathrm{Mg}$ II absorbers toward GRBs has proven a challenge.

In an effort to unveil this problem, Porciani et al. (2007) considered dust obscuration, beam size differences, intrinsic properties of GRBs, and gravitational lensing as possible causes. They found beam size differences irrelevant, with simulations predicting that the absorbing systems are significantly larger than either beam. Cucchiara et al. (2009) found no dissimilarities between the populations of absorbers, suggesting that it is unlikely that there are excess absorbers physically associated with the GRBs. This leaves dust obscuration of QSOs and gravitational lensing of GRBs as the two most plausible explanations.

Wyithe et al. (2011, hereafter W11) suggested that, being detected in two independent energy bands, GRBs could be subjected to a multi-band magnification bias (see Wyithe et al. 2003 for details). Consequently, observed GRBs could be more likely to be strongly gravitationally lensed than QSOs, with a resulting increase in absorber numbers due to the lensing galaxies. Following this prediction, Rapoport et al. (2012) investigated the probability distribution of alignment between GRBs with strong Mg II absorption and the galaxy closest to their LOS. The most interesting cases to suggest possible strong gravitational lensing scenarios were those of GRB020405, which showed a second transient $\sim 3^{\prime \prime}$ from the GRB's optical afterglow location, and GRB030429, which is aligned only 1".2 away from a massive galaxy that is known to have caused strong Mg II absorption.

As a part of their study of dusty systems along QSO LOS, Budzynski \& Hewett (2011, hereafter BH11) tested the bias in absorber numbers expected due to dust obscuration by foreground objects toward QSOs and concluded that while some bias is anticipated, it cannot solely account for the discrepancy. From the total sample of QSOs they studied, they found that $24 \% \pm 4 \%$ of strong $\mathrm{Mg}$ II absorbers are not observed because the QSOs are obscured and either fall below the typical signalto-noise $(\mathrm{S} / \mathrm{N})$ thresholds or are no longer detected.

In this paper we quantify the significance of the $\mathrm{Mg}$ II problem when including three new LOS, and describe the statistical tools we use for this purpose in Section 2. In Section 3, we analyze the gravitational lensing bias, presenting new observations of the lensing candidate, GRB020405; discuss the dust obscuration bias; and calculate the total bias required to resolve the discrepancy. Our conclusions are summarized and discussed in Section 4.

\section{SIGNIFICANCE OF THE Mg II PROBLEM}

To quantify the significance of the $\mathrm{Mg}$ II problem we require knowledge of the redshift path over which strong systems can be observed (also known as path density, $g(z)$ ) toward the GRBs. The appropriate comparison for the number of observed systems toward GRBs is the expected number of systems toward QSOs covering an equivalent $g(z)$, which is calculated from the QSO 
absorber number density $(\partial n / \partial z)$ as

$$
N_{\text {exp, QSO }}^{\mathrm{Mg}_{\text {II }}}=\int g(z) \frac{\partial n}{\partial z} d z .
$$

Recently, Lawther et al. (2012, hereafter L12) used the Sloan Digital Sky Survey (SDSS) DR7 to study QSOs with Mg II absorption lines. For each redshift bin they measured how many QSO spectra would allow detection of a strong Mg II system at that redshift, and the number of absorbers $N(z)$ observed in that bin. They find that for strong absorbers (equivalent width $(\mathrm{EW})>1 \AA$ ), the number density per comoving Mpc along an LOS, $n(X)$, can be well described as a function of redshift, $z$, by the following expression:

$$
c n(X) / H_{0}=n_{0} \exp \left(z_{0} / z\right)
$$

where $n_{0}=0.110 \pm 0.005$ absorbers per unit redshift and $z_{0}=-0.11 \pm 0.03$. The number density, $\partial n / \partial z$ is given by

$$
\frac{\partial n}{\partial z}=\frac{c n(X)}{H_{0}} \frac{(1+z)^{2}}{\sqrt{\Omega_{M}(1+z)^{3}+\Omega_{\Lambda}}} .
$$

In 2006, P06 reported that the probability of finding the number of strong Mg II absorbers observed toward 14 GRBs in a similar redshift path toward QSOs was $<0.1 \%$, using a Monte Carlo (MC) analysis. In 2009, V09 analyzed all GRBs with high-resolution spectra taken by UVES on the Very Large Telescope (VLT) up to 2008 June (10 objects). Their complete sample, which included 26 GRBs (16 with available data from the literature, including those from the P06 sample), revealed 22 strong Mg II systems. They found that for a similar redshift path toward QSOs, one would expect 10.41 strong absorbing systems. However, they incorrectly included Poisson statistics to express the error for this number when it was calculated from a fit to the absorber number density equation $(\partial n / \partial z)$ for QSOs. We advocate instead that it is best to use an MC approach to reflect the distribution of absorbers, such as was undertaken by $\mathrm{P} 06$.

Here, we revise the V09 work, deploying different statistical tools, and also include three new GRBs with high-resolution VLT data (GRB080804, GRB081008, and GRB081029).

\subsection{Additional Data}

We reduced and analyzed publicly available data for GRB080804, which we acquired from the ESO archive. The data were taken using the UVES instrument on the VLT, with a total $2.6 \mathrm{hr}$ of integration time. The data were acquired with a $1^{\prime \prime}$ slit in good seeing $\left(\sim 0^{\prime \prime} .7\right)$. The observations were reduced using the ESO Gasgano pipeline for UVES. ${ }^{4}$ The pipeline performs the standard tasks of bias subtraction, flat fielding, spectral order location, wavelength calibration, and extraction of the spectrum. Candidate $\mathrm{Mg}$ II doublets were identified by visual inspection of the spectra. The high $\mathrm{S} / \mathrm{N}$ of the data allowed lines exhibiting EWs as low as $0.1 \AA$ to be detected. All candidate doublets were then shifted to the rest frame to assess if the lines matched the Mg II doublet at 2794.4 $\AA$ and 2801.5 $\AA$. All EWs were measured in IRAF" using the "splot" task (Tody 1993). Any candidate $\mathrm{Mg}$ II doublets that appeared to be contaminated by other spectral features (e.g., absorption from other species

\footnotetext{
4 http://www.eso.org/projects/dfs/dfs-shared/web/vlt/vlt-instrumentpipelines.html

5 http://iraf.noao.edu
}

\begin{tabular}{|c|c|c|c|c|}
\hline GRB & $z_{\mathrm{GRB}}{ }^{\mathrm{a}}$ & $\Delta z^{\mathrm{b}}$ & $z_{\text {abs }}{ }^{\mathrm{c}}$ & Reference $^{\mathrm{d}}$ \\
\hline 991216 & 1.022 & 0.636 & $\begin{array}{l}0.770 \\
0.803\end{array}$ & 1 \\
\hline 000926 & 2.038 & 1.392 & $\ldots$ & 2 \\
\hline 010222 & 1.477 & 1.022 & $\begin{array}{l}0.927 \\
1.156\end{array}$ & 3 \\
\hline 011211 & 2.142 & 1.566 & $\ldots$ & 1 \\
\hline 020405 & 0.695 & 0.312 & 0.472 & 4 \\
\hline 020813 & 1.255 & 0.866 & 1.224 & 5 \\
\hline 021004 & 2.3295 & 1.756 & $\begin{array}{l}1.3800 \\
1.6026\end{array}$ & 6 \\
\hline 030226 & 1.986 & 1.590 & $\ldots$ & 7 \\
\hline 030323 & 3.372 & 0.822 & $\ldots$ & 8 \\
\hline 030328 & 1.522 & 1.131 & $\ldots$ & 9 \\
\hline 030429 & 2.66 & 1.241 & 0.8418 & 10 \\
\hline 050505 & 4.275 & 0.856 & $\begin{array}{l}1.695 \\
2.265\end{array}$ & 11 \\
\hline 050730 & 3.9687 & 1.298 & $\ldots$ & 6 \\
\hline 050820A & 2.6147 & 1.845 & $\begin{array}{l}0.6915 \\
1.4288\end{array}$ & 6 \\
\hline 050908 & 3.35 & 1.456 & 1.548 & 12 \\
\hline $050922 \mathrm{C}$ & 2.1996 & 1.682 & $\ldots$ & 6 \\
\hline 051111 & 1.55 & 1.036 & 1.19 & 12 \\
\hline 060206 & 4.048 & 1.060 & 2.26 & 13,14 \\
\hline 060418 & 1.4900 & 1.265 & $\begin{array}{l}0.6026 \\
0.6559 \\
1.1070\end{array}$ & 6 \\
\hline 060526 & 3.221 & 1.434 & $\ldots$ & 15 \\
\hline 060607A & 3.0748 & 1.713 & 1.8033 & 6 \\
\hline 071003 & 1.604 & 1.212 & 0.372 & 16 \\
\hline 071031 & 2.6922 & 1.789 & $\ldots$ & 6 \\
\hline 080310 & 2.4272 & 1.841 & $\ldots$ & 6 \\
\hline 080319B & 0.9378 & 0.57 & 0.7154 & 6 \\
\hline 080413A & 2.4346 & 1.650 & $\ldots$ & 6 \\
\hline 080804 & 2.20 & 1.63 & $\ldots$ & This work \\
\hline 081008 & 1.286 & 0.92 & $\ldots$ & 17 \\
\hline 081029 & 3.8479 & 1.50 & $\ldots$ & 18 \\
\hline
\end{tabular}

Table 1

GRB Sample

Notes.

a Redshift of GRB.

${ }^{\mathrm{b}}$ Redshift path length for strong Mg II absorbers.

c Strong absorber redshift.

d References. (1) Vreeswijk et al. 2006; (2) Castro et al. 2003; (3) Mirabal et al. 2002; (4) Masetti et al. 2003; (5) Barth et al. 2003; (6) Vergani et al. 2009; (7) Klose et al. 2004; (8) Vreeswijk et al. 2004; (9) Maiorano et al. 2006; (10) Jakobsson et al. 2004; (11) Berger et al. 2006; (12) Prochter et al. 2006b; (13) Chen et al. 2009; (14) Hao et al. 2007; (15) Thöne et al. 2010; (16) Perley et al. 2008; (17) D'Elia et al. 2011; (18) Holland et al. 2012.

at the GRB redshift, or other foreground absorption lines) were deblended in IRAF with the splot task. None of the absorption features were deep enough to have obscured the presence of a strong absorption doublet having the wavelength ratio of $\mathrm{Mg}$ II. The total wavelength coverage is 3600-9000 $\AA$ with telluric features preventing possible identification of the doublet between

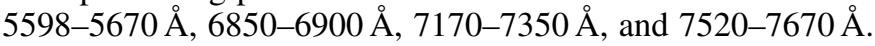
The wavelength limits were converted into redshift space for inclusion in the redshift path density after placing a lower limit using the Ly $\alpha$ break, and an upper one at the GRB redshift. No strong Mg II absorbing system was identified.

The redshift path and absorber information for GRBs 081008 and 081029 were taken from the literature (D'Elia et al. 2011; Holland et al. 2012, respectively) and were corrected for telluric features. The full data set is listed in Table 1, and the corresponding redshift path density is shown in Figure 1. 


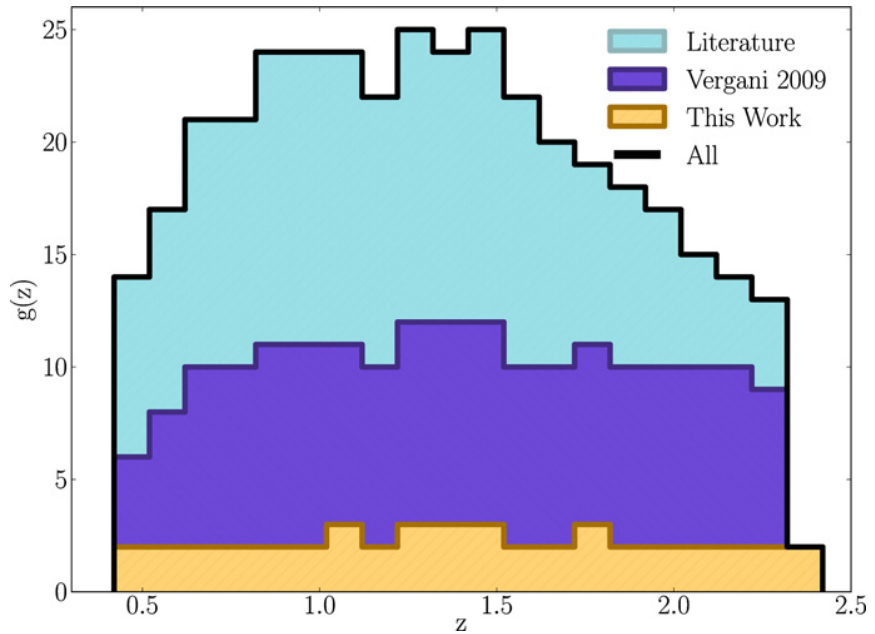

Figure 1. Redshift path density $g(z)$ for absorbers with EW $>1 \AA$ toward the GRBs used in this study. The contribution labeled "Vergani 2009" includes only the 10 UVES GRBs, while the values from the literature were also included in the full sample in V09. The objects we added to the study are shown in yellow. The total sample used in the paper is the sum of all the different contributions and is outlined with the black curve.

(A color version of this figure is available in the online journal.)

\subsection{Statistical Analysis}

We calculate the number of strong $\mathrm{Mg}$ II absorbers one would expect to find toward QSOs for the redshift path of our observed GRBs using Equation (1). Using the V09 redshift path density for only the 10 UVES GRBs, which included nine strong absorbing systems, L12 predicted that $4.1_{-0.7}^{+0.8}$ strong absorption systems would be expected from a similar QSO sample. While the relatively small errors represent the degree of precision in the mean expected value for strong absorbers along QSOs LOS, we note that the probability around the mean value is not a Gaussian distribution, and therefore that the error cannot be used to directly estimate the significance of the $\mathrm{Mg}$ II problem.

Using $\partial n / \partial z$ from L12, and the $g(z)$ for our 29 GRBs, we find $N_{\text {exp }}^{\mathrm{Mg} \text { II }}=9.6 \pm 0.7$ for a comparable sample of QSO LOS, where the errors are propagated from the uncertainties of the fitting parameters for $\partial n / \partial z\left(n_{0}\right.$ and $\left.z_{0}\right)$. When using the P06 values for $n_{0}$ and $z_{0}$ (values found for SDSS DR3), $N_{\text {exp,QSO }}^{\mathrm{Mg} \text { II }}=7.4 \pm 0.7$ with the $g(z)$ provided in V09 only, and $8.3 \pm 0.8$ for the $g(z)$ included in this work. The difference between the GRB and the mean of the QSO populations is reduced when using our sample relative to the earlier V09 sample. It is further reduced when using the updated L12 fit instead of the P06 fit to the QSO number density (as the expected value for QSO absorbers increases). Thus, as more data are available, both for GRBs and QSOs, the difference between the expected number of absorbers toward QSOs and the observed population toward GRBs is seen to decline, implying that we are still strongly affected by small number statistics.

In order to assess the significance of the current $\mathrm{Mg}$ II problem we calculate how unlikely it would be to observe 22 strong Mg II systems along the LOS to 29 GRBs, under the hypothesis that GRBs and QSOs probe the same absorber population. We use the SDSS DR4 catalog of Quider et al. (2011), which includes 44,600 QSOs spectra and contains $\sim 17,000$ measured $\mathrm{Mg}$ II doublets. The catalog provides information regarding the redshift of the QSO and the redshift and EW of the $\mathrm{Mg}$ II absorber. We conduct an MC simulation in which we randomly select QSOs from the sample with similar $g(z)$ to the observed
GRBs, and count the number of strong absorbers toward them. We are not constrained to match the precise number of source systems, as the absorber rate depends only on the redshift path being probed. Therefore, for each redshift bin of the $g(z)$ function (see Figure 1) we select QSOs at a redshift of a randomly selected GRB (within a 0.1 redshift bin), constraining the GRB sample to those at a redshift that is larger than the bin, and small enough so the tested redshift bin does not fall shortward of the Ly $\alpha$ break in the QSO spectrum. Once an appropriate random QSO is chosen, we count the number of absorbers within the redshift bin. We continue this procedure until we have covered the full redshift path of our $g(z)$ function. Our MC analysis includes 10,000 trials of the full path selection.

Our analysis reproduces the mean value calculated above from the empirical fits of P06 (8.3 absorbers). The histogram of the number of absorbers is consistent with a Gaussian distribution, for which we measure a standard deviation of $\sigma=3.2$ absorbers. Our simulation resembles the results of P06 more than those of L12 as we are using the QSO Mg II absorbers catalog from SDSS DR4. The SDSS DR4 is the largest publicly available catalog that includes all the data required to conduct this analysis. P06 found fewer absorbers per unit redshift in the DR3 sample than the L12 DR7 study, as seen in Figure 3 of L12. The observation of 22 absorbers among a population with a mean of 8.3 and $\sigma=3.2$ would be $3.9 \sigma$ from the average value. As expected if the distribution is Gaussian, we find no instances of a sample with 22 or more strong absorbers in our $10,000 \mathrm{MC}$ trails. To test the sensitivity of our results to the chosen QSO redshift we repeat the MC analysis and do not constrain the QSOs to have similar redshifts as the GRBs. We do not find any statistically significant deviations in the results, either in the absorber numbers or in the redshift distribution of the absorbing systems. Moreover, the redshifts of the absorbing systems are similar between the QSOs and the GRBs.

\section{POTENTIAL BIAS CONTRIBUTIONS}

As the difference between the GRB and QSO LOS is statistically significant, we now explore the two leading explanations for this discrepancy: gravitational lensing bias and dust obscuration bias.

\subsection{Gravitational Lensing Bias}

A potential contributor to the $\mathrm{Mg}$ II problem is that GRBs with observed afterglows are more likely to be gravitationally lensed than QSOs (see W11 and references therein). This is attributed to the multi-band magnification bias arising from GRBs being detected in two bands ( $\gamma$-rays and optical) with uncorrelated intrinsic fluxes (see Wyithe et al. 2003 for details). If GRBs are preferentially lensed compared to QSOs, a higher rate of $\mathrm{Mg}$ II absorption would be expected toward GRBs from the gaseous halos surrounding the lensing galaxies.

\subsubsection{GRB020405}

Following the W11 prediction that if gravitational lensing bias is the explanation for the Mg II problem, then $10 \%-60 \%$ of GRBs with strong Mg II absorption should have been lensed strongly enough to produce multiple images, we studied archival data of the GRBs from the V09 sample to look for potential strongly gravitationally lensed systems (Rapoport et al. 2012). Two potential cases of lensing were identified, one of which was GRB020405 $(z=0.695)$, which had a nearby $\left(3^{\prime \prime}\right)$ transient that could have been a repeating image of the same GRB. 

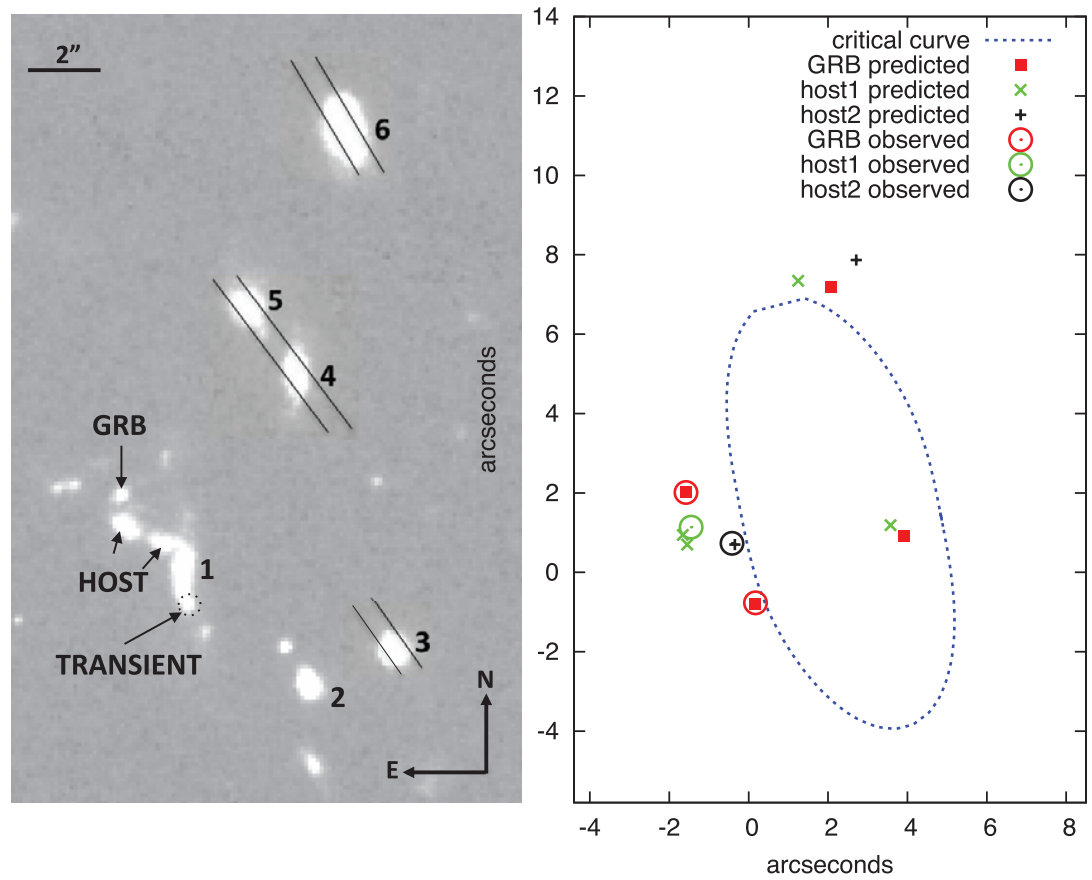

Figure 2. Left: HST WFPC2/F702W field of GRB020405. The GRB is clearly visible and the complex host and second transient are indicated. Objects 1 and 2 were found to be at the strong absorber's redshift of $z=0.472$. Our observations indicate that object 3 is a field star, galaxy 4 is a galaxy at $z=0.485 \pm 0.002$, object 5 has unknown redshift, and galaxy 6 is at $z=0.484 \pm 0.002$. The slits used in the Gemini/GMOS observations are superimposed on the image. Right: LENSMODEL solution suggested in Rapoport et al. (2012), which assumed objects 1-6 were at the strong absorber redshift of $z=0.472$. With the newly measured redshifts of objects 4 and 6, we are not able to identify any gravitational lensing model to explain the transient as another image of the GRB.

(A color version of this figure is available in the online journal.)

The transient near GRB020405 was first observed by Masetti et al. (2003) in a Hubble Space Telescope (HST) image taken $\sim 19$ days after the Swift trigger. They noted that it was not visible in F555W but only in F702W and F814W, and had completely faded by the last observation in 2002 August. Figure 2 shows the locations of the GRB, transient, and host galaxy. Objects 1 and 2 were confirmed, using VLT spectroscopy, to be at the strong absorber redshift $(z=0.472)$. Using GRAVLENS (Keeton 2001) to model the system, we found that if objects 3-6 (or even only 4-6) are part of a group at the absorbers' redshift, the observations could be explained as a multiply imaged GRB (see Rapoport et al. 2012 for details and the right panel of Figure 2 for a potential model). In order to test this scenario we initiated a detailed analysis of the surrounding galaxies.

We obtained spectra for several of the galaxies around GRB020405 using the GMOS instrument (Hook et al. 2004) on Gemini-South (PI: Rapoport, Program ID GS-2012A-Q-9). The multi-slit data were taken on UT 2012 April 3 and consisted of eight $120 \mathrm{~s}$ exposures with the R150 grating. The $1^{\prime \prime}$ slit widths provided a spectral resolution of $R \sim 315$, and the data were binned $4 \times 4$ to yield a spatial scale of $\sim 0^{\prime} .3$ pixel $^{-1}$ and a dispersion of $13.6 \AA \mathrm{pixel}^{-1}$. To improve the removal of sky and fringing features, the mask was cut with two sets of slits, offset by $85^{\prime \prime}$, and the field was dithered between the two positions. The data were reduced using the standard Gemini/IRAF packages ${ }^{6}$ for flat fielding and wavelength solution. No standard flux correction was applied as the only information needed from the observations were redshifts.

Comparing our reduced spectra to a library of templates using the RUNZ 2dFGRS redshift code (R. Sharp 2012, private

\footnotetext{
6 http://www.gemini.edu/sciops/data-and-results/processing-software
}

communication; see Figure 3), we were able to determine that objects 4 and 6 are galaxies at $z=0.485 \pm 0.002$ and $z=0.484 \pm 0.002$, respectively, where the errors on the redshifts are based on a centroid fit to the strongest line. Object 3 is consistent with being a foreground star. The spectra for object 5 were not of sufficient $\mathrm{S} / \mathrm{N}$ to determine the type or redshift of the object.

As the galaxies at $z=0.472$ and $z=0.484$ are too distant to be from the same halo $\left(\Delta v=4800 \mathrm{~km} \mathrm{~s}^{-1}\right)$, but objects 4 and 6 are at a similar redshift, we modeled the field using two different groups (objects 1 and 2, and objects 4 and 6). In order to simplify the mathematics we approximated the two groups (each as a singular isothermal ellipse with shear) to reside in one plane at the strong absorber redshift $(z=0.472)$. No model was found that could explain the observables using these galaxy redshifts and allowing plausible masses for the galaxies. The velocity dispersion was limited to $800 \mathrm{~km} \mathrm{~s}^{-1}$ for each galaxy group, which is beyond the expected value of $100-500 \mathrm{~km} \mathrm{~s}^{-1}$ found for groups and poor clusters (Bahcall 1995).

Therefore, we conclude that the transient near GRB020405 was not another image of that GRB, leaving one potential candidate as a strongly gravitationally lensed GRB from the sample studied in our previous work, GRB030429. This GRB was detected 1".2 away from a strong Mg II absorbing spiral galaxy. Using photometric observations, a spectral energy distribution fitting technique implied $M_{B}=-21.1 \pm 0.1$ for the absorbing galaxy. The Tully-Fisher relation for such an intrinsically bright galaxy suggests a velocity dispersion of $160 \pm 65 \mathrm{~km} \mathrm{~s}^{-1}$, where $200 \mathrm{~km} \mathrm{~s}^{-1}$ is required for producing a second image for this GRB (see Rapoport et al. 2012 for further details). Thus, GRB030429 is likely to be magnified at some level, possibly strongly. Verifying this scenario would require measuring the velocity dispersion of the galaxy, which, with 


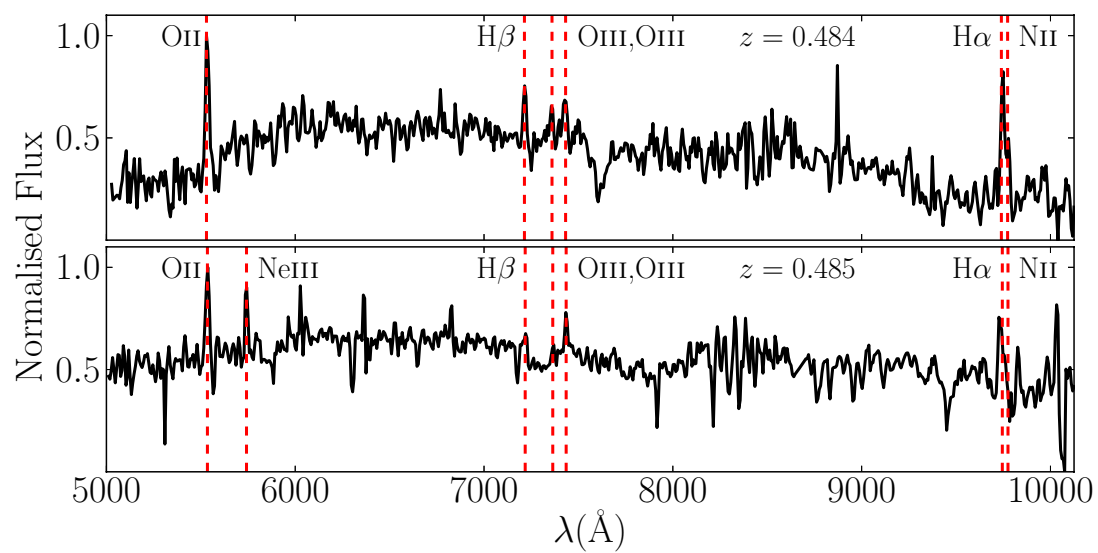

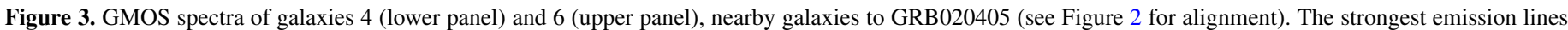
are identified and find galaxy 4 to be at $z=0.485 \pm 0.002$ and galaxy 6 at $z=0.484 \pm 0.002$.

(A color version of this figure is available in the online journal.)

$R=22.70 \pm 0.12 \mathrm{mag}$ (Jakobsson et al. 2004) and at a redshift of 0.841 , would be observationally very expensive.

In our previous analysis of the existing HST imaging data for $11 \mathrm{GRBs}$ with $\mathrm{Mg}$ II absorption from the V09 sample, we ruled out strong gravitational lensing in 6 cases. Now having determined that GRB020405 was not strongly lensed, we can place an upper $(2 \sigma)$ limit on the fraction of strong absorbers which lead to multiply imaged GRB of $\lesssim 35 \%$, though this is a very conservative limit as the remaining systems show no evidence of lensing. Assuming only the most likely case of GRB030429 is a viable strongly gravitationally lensed candidate, the lensing fraction is reduced to $\lesssim 13 \%(2 \sigma)$.

\subsubsection{Statistical Analysis}

To test the effect of strong gravitational lensing on the $\mathrm{Mg}$ II absorber statistics, we account for the fraction of strongly gravitationally lensed GRBs $\left(F_{\text {lens }}\right)$ by multiplying the number of absorbers found in the MC trials by $1 /\left(1-F_{\text {lens }}\right)$. This approach assumes that all strongly gravitationally lensed objects would show strong Mg II absorption. We find the probability of finding 22 absorbers for a lensing fraction of 0.05 (assuming the Mg II absorber toward GRB030429 was the only one out of the 22 systems that caused strong lensing) to be $0.02 \%$. Moreover, even if all four remaining candidates from Rapoport et al. (2012) were strongly gravitationally lensed, the probability of observing 22 absorbers would only increase to $0.7 \%$. This indicates that lensing alone cannot account for the $\mathrm{Mg}$ II problem.

\subsection{Dust Obscuration Bias}

The Mg II problem could also arise by flux-limited QSO samples selecting against absorbed objects with high Mg II EW. Among a QSO sample having the same redshift path density as GRBs in the V09 sample, BH11 showed that $20 \%$ (see Figure 19 in BH11) of sources with strong absorbing systems would have been obscured by dust and so either not detected or below the $\mathrm{S} / \mathrm{N}$ threshold (for EW $>1 \AA$ ). ${ }^{7}$ However, previous work by Sudilovsky et al. (2009, hereafter S09) found that dust can only explain $\approx 10 \%$ of the discrepancy. The different

\footnotetext{
7 At higher redshift one would expect the QSOs to appear fainter and be more susceptible to dust obscuration. As the added $g(z)$ in this work, relative to that in V09, is flat (see Figure 1), the redshift path could be thought as being more concentrated at higher redshifts than it is in the V09 sample. Therefore, by using the value found by BH11 for the percentage of obscured systems, we are more likely to underestimate the number of obscured QSOs than not.
}

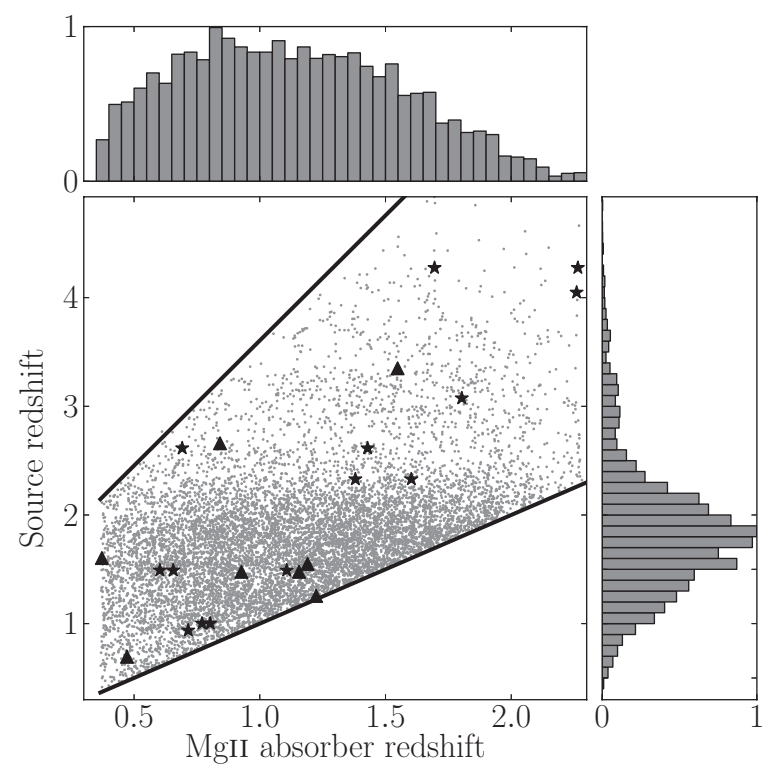

Figure 4. Redshifts of the QSOs, GRBs, and their strong absorbing systems. The Quider et al. (2011) QSO sample used in the MC analysis is represented by gray points and their corresponding normalized histograms. The black triangles and the black stars mark the GRBs with absorbers from the full sample used in this paper, where the stars differentiate those GRBs that would have been excluded in the dust obscuration analysis of BH11. That is, GRBs with more than two absorbing systems, GRBs at redshifts larger than 3.5, and GRBs with a $>5 \AA$ absorbing system. The lower black line represents the $z_{\mathrm{Mg} \text { II }}=z_{\mathrm{QSO}}$ boundary and the upper black line is the limit due to Ly $\alpha$ absorption.

conclusions between these two studies are attributable to several causes. S09 limited their QSO sample to those at $z<2.0$ while the BH11 limit was set at $z<3.5$ which better matches the redshifts of the GRBs in this study. Moreover, S09 used $d n / d z$, the number density of $\mathrm{Mg}$ II absorbers, throughout their analysis. Here we argue that the quantity $d n / d z$ is a less useful measure of the discrepancy as it does not include information on the specific redshift paths ranges, but only the total redshift path coverage. As the upper panel of Figure 4 shows, the absorbers are not evenly distributed in redshift space. Therefore, the probability of finding a strong absorber must depend on the specific redshift range at which a system can be detected, and not only on the redshift path length. Furthermore, as has been discussed in Section 3.1.2, newer SDSS catalogs reveal stronger absorbers along QSOs LOS and the two studies were performed using 


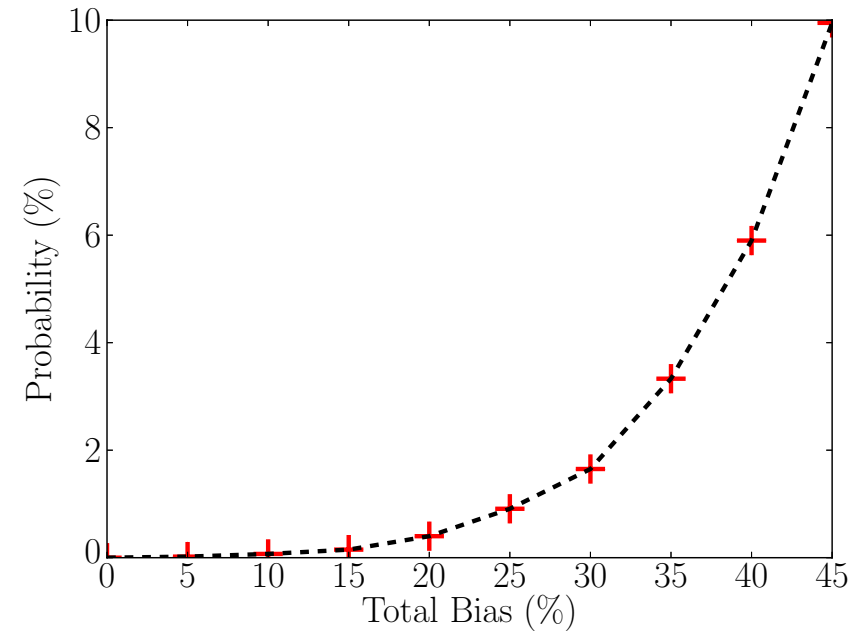

Figure 5. Probability of finding 22 strong absorbing systems along the LOS to QSOs having a similar redshift path density to that of the GRB sample for different fractions of total bias. The number of absorbers found along the QSOs LOS in the MC is multiplied by $1 /\left(1-F_{\mathrm{Bias}}\right)$ to account for the missing fraction before being compared to the number of absorbers found toward GRBs.

(A color version of this figure is available in the online journal.)

different SDSS data releases, with S09 using DR5 and BH11 using DR7. Finally, it is important to note that both BH11 and S09 declare their results should be considered as lower limits.

Thus, using the most recent $\mathrm{BH} 11$ results, the QSO sample is likely missing $20 \%$ of the strong absorbing systems. To correct for this dust obscuration, we multiply the predicted absorber rate from our MC analysis by a factor of $1 /(1-0.2)$. This correction results in a mean of 10.8 absorbers, with a probability of finding 22 absorbers of $0.4 \%$.

\subsection{Total Bias}

As neither dust obscuration nor gravitational lensing by themselves can explain the $\mathrm{Mg}$ II problem, we repeat the $\mathrm{MC}$ simulation and investigate the total bias required to resolve the difference. We do this by imposing a bias on the QSOs by multiplying the number of absorbers by $1 /\left(1-F_{\text {Bias }}\right)$. We then increment the bias fraction $\left(F_{\text {Bias }}\right)$ until it can account for the observed excess toward GRBs. We find that for a total bias fraction of $\sim 30 \%(45 \%)$ the significance of the problem is reduced to $2 \sigma(1 \sigma)$ (see Figure 5). This formalism assumes that the majority of the bias arises from the QSOs, which is consistent with the $\sim 20 \%$ bias estimated by BH11.

BH11 noted that because their sample is flux limited they are likely to underestimate the fraction of absorbers missing from the SDSS DR7, with systems having EW > $5.0 \AA$ being completely removed. Moreover, in their analysis they only use QSOs up to a redshift of 3.5 and limit the number of absorbers per LOS to two. Figure 4 shows the QSO, GRB, and absorber redshifts used in our MC analysis. To demonstrate the effect BH11's QSOs selection would have on the GRB sample, we flag the GRBs that would have been omitted from the BH11 sample with the star-like markers. That is, GRBs with more than two absorbing systems, GRBs at redshifts larger than 3.5, and GRBs with a $>5 \AA$ absorbing system. The large fraction $(60 \%)$ of absorbers along the LOS to GRBs that would have statistically gone through dustier systems than the QSOs that were examined by BH11 illustrates that the dust obscuration bias could be underestimated. Therefore, while strong gravitational lensing might be playing a minor role, the likelihood that the dust obscuration bias has been underestimated implies that this effect can more easily resolve the $\mathrm{Mg}$ II problem.

\section{DISCUSSION}

We have presented an updated analysis of the GRB strong $\mathrm{Mg}$ II absorber problem, including additional GRB LOS. We outline a statistical method with which the $\mathrm{Mg}$ II problem can be addressed, using an $\mathrm{MC}$ technique to estimate the number of absorbers expected toward QSOs for an observed redshift path toward GRBs. We find that the observed discrepancy between the number of strong $\mathrm{Mg}$ II absorbers in GRBs and QSOs has a level of $4 \sigma$ significance, with $<0.01 \%$ chance of finding the observed number of absorbers along GRB LOS in the MC results for the QSOs.

Gravitational lensing bias for GRBs can contribute to the discrepancy, and we examined the best lensing candidate of Rapoport et al. (2012), GRB020405, which has a known foreground absorber and a second nearby transient. We find that the nearby galaxies, which would need to be at the absorber's redshift to allow possible strong gravitational lensing by a galaxy group, are not at the appropriate redshift. With no feasible lensing model found for the GRB and its nearby transient, we conclude that GRB020405 was not affected by strong lensing. However, there remains the possibility that gravitational lensing is playing a role, as evidenced by the very close alignment of GRB030429 to a massive foreground galaxy. If this GRB is significantly lensed, then the Mg II discrepancy is slightly reduced, with the probability of finding the number of strong absorbers along the GRBs increasing to $0.02 \%$. If four GRBs from the sample studied by Rapoport et al. (2012) are strongly lensed, this implies a lensing fraction of $20 \%$, which would increase the probability of finding the observed number of absorbers in a comparable QSO sample to only $0.7 \%$. Thus we conclude that lensing is very unlikely to solve the $\mathrm{Mg}$ II problem on its own.

The significance of the problem is also reduced after accounting for dust obscuration bias, with the probability of finding the observed number of absorbers along the LOS to the GRBs increasing to $0.4 \%$. When including both dust and gravitational lensing biases, we calculate a probability of $0.9 \%$, which remains statistically significant. In order to reduce the problem to below a $2 \sigma$ level, a total bias of $\sim 30 \%$ is required. We suggest this could be largely satisfied by the dust obscuration bias toward QSOs currently being underestimated.

If the main contributor to the discrepancy is dust, one would expect GRBs with strong absorbers to be heavily reddened or obscured. As gamma rays are not subjected to dust obscuration, a lack of detection in the optical might be the result of a blocking system. In order to find these obscured GRBs, a detailed deep imaging campaign within the error circle of GRBs needs to take place. The discovery of nearby massive galaxies could suggest obscuration prevented the detection of the optical afterglow.

Measuring the reddening of GRBs with strong absorbers is also challenging. As GRBs' spectra are expected to follow a power law, the common technique for correcting for reddening includes a correction for the galactic extinction plus a best-fit result for the host's extinction. As the host's extinction is found by matching the observed spectra to a power law, any inclusion of another reddening system along the LOS is redundant. A better fit will always be found when adding additional parameters, such as an absorbing system along the LOS. A good example for this is found in Ellison et al. (2006) where 
they clearly identify the $2175 \AA$ bump caused by an intervening system, suggesting that strong Mg II absorbers indeed result in some dust obscuration and reddening.

Following the submission of our paper, Cucchiara et al. (2012) increased the sample of GRBs to 118 LOS by including GRBs with low-resolution spectra. This study has found the rate of Mg II absorbers to GRBs to be lower than previous studies, reducing the overall discrepancy between the absorber rate to GRBs and QSOs to $<90 \%$ confidence level. If additionally one were to include the dust obscuration bias described above, we expect the rate of absorbers between GRBs and QSOs to be completely consistent.

S.R. gratefully acknowledges Robert Sharp for assistance with the redshift identification of the Gemini galaxies, Stuart Sim for useful conversation, and the anonymous referee for invaluable comments. B.P.S. acknowledges financial support through an ARC Laureate Fellowship Grant FL0992131. J.S.B.W. acknowledges financial support through an ARC Laureate Fellowship. A.O.T. acknowledges support from Sonderforschungsbereich SFB 881 "The Milky Way System" (subproject A5) of the German Research Foundation (DFG). This work is based on observations obtained at the Gemini Observatory, which is operated by the Association of Universities for Research in Astronomy, Inc., under a cooperative agreement with the NSF on behalf of the Gemini partnership: the National Science Foundation (United States), the Science and Technology Facilities Council (United Kingdom), the National Research Council (Canada), CONICYT (Chile), the Australian Research Council (Australia), Ministério da Ciência, Tecnologia e Inovação (Brazil), and Ministerio de Ciencia, Tecnología e Innovación Productiva (Argentina).

\section{REFERENCES}

Bahcall, N. A. 1995, in AIP Conf. Ser. 336, Dark Matter, ed. S. S. Holt \& C. L. Bennett (Melville, NY: AIP), 201

Barth, A. J., Sari, R., Cohen, M. H., et al. 2003, ApJL, 584, L47

Berger, E., Penprase, B. E., Cenko, S. B., et al. 2006, ApJ, 642, 979

Budzynski, J. M., \& Hewett, P. C. 2011, MNRAS, 416, 1871

Castro, S., Galama, T. J., Harrison, F. A., et al. 2003, ApJ, 586, 128

Chen, H.-W., Perley, D. A., Pollack, L. K., et al. 2009, ApJ, 691, 152

Cucchiara, A., Jones, T., Charlton, J. C., et al. 2009, ApJ, 697, 345

Cucchiara, A., Prochaska, J. X., Zhu, G., et al. 2012, arXiv:1211.6528

D’Elia, V., Campana, S., Covino, S., et al. 2011, MNRAS, 418, 680

Ellison, S. L., Vreeswijk, P., Ledoux, C., et al. 2006, MNRAS, 372, L38

Hao, H., Stanek, K. Z., Dobrzycki, A., et al. 2007, ApJL, 659, L99

Holland, S. T., De Pasquale, M., Mao, J., et al. 2012, ApJ, 745, 41

Hook, I. M., Jørgensen, I., Allington-Smith, J. R., et al. 2004, PASP, 116, 425

Jakobsson, P., Hjorth, J., Fynbo, J. P. U., et al. 2004, A\&A, 427, 785

Keeton, C. R. 2001, arXiv:astro-ph/0102340

Klose, S., Greiner, J., Rau, A., et al. 2004, AJ, 128, 1942

Lawther, D., Paarup, T., Schmidt, M., et al. 2012, A\&A, 546, A67

Maiorano, E., Masetti, N., Palazzi, E., et al. 2006, A\&A, 455, 423

Masetti, N., Palazzi, E., Pian, E., et al. 2003, A\&A, 404, 465

Mirabal, N., Halpern, J. P., Kulkarni, S. R., et al. 2002, ApJ, 578, 818

Perley, D. A., Li, W., Chornock, R., et al. 2008, ApJ, 688, 470

Porciani, C., Viel, M., \& Lilly, S. J. 2007, ApJ, 659, 218

Prochter, G. E., Prochaska, J. X., \& Burles, S. M. 2006a, ApJ, 639, 766

Prochter, G. E., Prochaska, J. X., Chen, H.-W., et al. 2006b, ApJL, 648, L93

Quider, A. M., Nestor, D. B., Turnshek, D. A., et al. 2011, AJ, 141, 137

Rapoport, S., Onken, C. A., Schmidt, B. P., et al. 2012, ApJ, 754, 139

Sudilovsky, V., Smith, D., \& Savaglio, S. 2009, ApJ, 699, 56

Thöne, C. C., Kann, D. A., Jóhannesson, G., et al. 2010, A\&A, 523, A70

Tody, D. 1993, in ASP Conf. Ser. 52, Astronomical Data Analysis Software and Systems II, ed. R. J. Hanisch, R. J. V. Brissenden, \& J. Barnes (San Francisco, CA: ASP), 173

Vergani, S. D., Petitjean, P., Ledoux, C., et al. 2009, A\&A, 503, 771

Vreeswijk, P. M., Ellison, S. L., Ledoux, C., et al. 2004, A\&A, 419, 927

Vreeswijk, P. M., Smette, A., Fruchter, A. S., et al. 2006, A\&A, 447, 145

Wyithe, J. S. B., Oh, S. P., \& Pindor, B. 2011, MNRAS, 414, 209

Wyithe, J. S. B., Winn, J. N., \& Rusin, D. 2003, ApJ, 583, 58 